\title{
Open radical retropubic prostatectomy as a saviour in the era of robotics- a single surgeon experience
}

\author{
Abdul Rouf Khawaja ${ }^{1}$, Malik Abdul Rouf ${ }^{1}$, Yasir Dar ${ }^{1}$, Tariq Malik ${ }^{2}$, Khalid Sofi ${ }^{3}$, Javeed Magray ${ }^{1}$, Sajad Malik $^{1}$, Arif \\ Hamid Bhat ${ }^{1}$, Mohd.Saleem Wani ${ }^{1}$
}

Cite this article: Khawaja AR, Rouf MA, Dar Y, Sofi K, Magray J et al: Open Radical Retropubic Prostatectomy as a Saviour in the Era of Robotics- A Single Surgeon Experience. Ann Urol Oncol 2020; 3(2): 54-60. https://doi. org/10.32948/auo.2020.10.08

\begin{abstract}
Background Outcome of its safety, perioperative outcomes, functional outcomes, oncologic outcomes in open radical retropubic prostatectomy for organ confined prostate cancers in the era of robotic surgery.

Methods A prospective study of radical retropubic prostatectomy performed at SKIMS between 2013 and 2020 was conducted. Work up of the patients in the study $(n=42)$ included age, comorbidities, serum prostate-specific antigen levels, digital rectal examination, MPMRI prostate, prostatic biopsy (Gleasons score), bone scan and optional PSMA PET scan. Intraoperative findings and pathological variables -T stage, nodal status, any extraprostatic extension, apical margin, bladder neck, seminal vesical invasion, lymph nodal status, post operative BCR need for any hormonal and salvage radiotherapy were recorded. On follow up particular emphasis was given on trifecta as cancer control, urinary continence, erectile function and overall satisfaction.

Results Out of 42 patients 7 patients were continent at 1 month follow up, 27 at 3 months, 39 at 6 months and 41 at 12 months. One patient continued to be incontinent at 1 year. Out of 16 patients with nerve sparing RPP 10 patients were potent with PD 5 inhibitor assistance at 6 months and all at 1 year ( Potency was defined as the ability to have erections adequate enough for penetration more than $50 \%$ of the times). Three patients had Biochemical recurrence on follow up and both were subjected to hormonal and salvage radiotherapy. Thirty nine patients were disease free at last follow up.

Conclusions Radical prostatectomy is the standard of care for organ confined prostatic carcinoma. Aim of the procedure is trifecta as cancer control, urinary continence, and erectile function. Minimally invasive techniques as Robotics should not be a limiting factor especially when affordablity and non availability is concern.
\end{abstract}

Key words Organ confined prostatic carcinoma, open radical retropubic prostatectomy, robotassisted radical prostatectomy, biochemical recurrence

1. Department of Urology, Sheri Kashmir Institute of Medical Sciences, Srinagar Soura Jammu and Kashmir 190011, India.

2. Department of Radiotherapy,Sheri Kashmir Institute of Medical Sciences,Srinagar Soura Jammu and Kashmir 190011, India.

3. Department of Anaesthesia, Sheri Kashmir Institute of Medical Sciences, Srinagar Soura Jammu and Kashmir 190011, India.

Correspondence: Abdul Rouf Khawaja Uro oncologist (Department of Urology, Sheri Kashmir Institute of Medical Sciences, Srinagar Soura Jammu and

Kashmir 190011, India; Email: roufkhawaja@rediffmail.com). 


\section{Introduction}

Prostate cancer is a major health problem world wide and is the second most frequently diagnosed cancer in men worldwide and the fifth most common cancer overall [1]. It accounts for nearly $21 \%$ of all newly diagnosed cancers in male. For reasons that remain unclear, the risk of prostate cancer is $70 \%$ higher in blacks as compare to whites [2]. It is also the sixth leading cause of cancer deaths in men. Globally, prostate cancer is projected to have the largest proportionate increase in cancer cases in men by 2020 .

Widespread PSA screening has led to higher rate of diagnosis of clinically localized prostate cancer in young and healthier men, resulting in more longevity after the treatment [3]. Treatment strategies used for treating localized prostate cancer with curative intent such are radical prostatectomy, radiotherapy, cryotherapy or HIFU. During the radical prostatectomy, the entire prostate gland is removed.

Radical prostatectomy (RP) is a standard treatment for localized prostate cancer. The open radical prostatectomy with a retropubic approach was gold standard for many years. The first radical prostatectomy was performed by the perineal approach in 1903 by Young [4] and the first retropubic approach described by Millin [5] in 1947. The current concept of retropubic radical nerve-sparing prostatectomy was established by Patrick Walsh et al. in 1982 [6] The next step in the evolution of open radical prostatectomies was transfer to a field of minimally invasive surgeries, i.e. laparoscopic radical prostatectomy (LRP) and robot-assisted laparoscopic radical.

Oncological outcomes and positive margin rates (PMR) are equivalent between robot-assisted radical prostatectomy (RARP) and radical retropubic prostatectomy (RRP) [7].

High cost and lack of widespread availability are the limitatations with robot-assisted radical prostatectomy there by making the open surgery a viable option especially in developing countries. In this study, we assessed the safety, perioperative outcomes,functional outcomes, oncological outcomes, with specific emphasis on trifecta and overall satisfaction after RPP.

\section{Materials and methods}

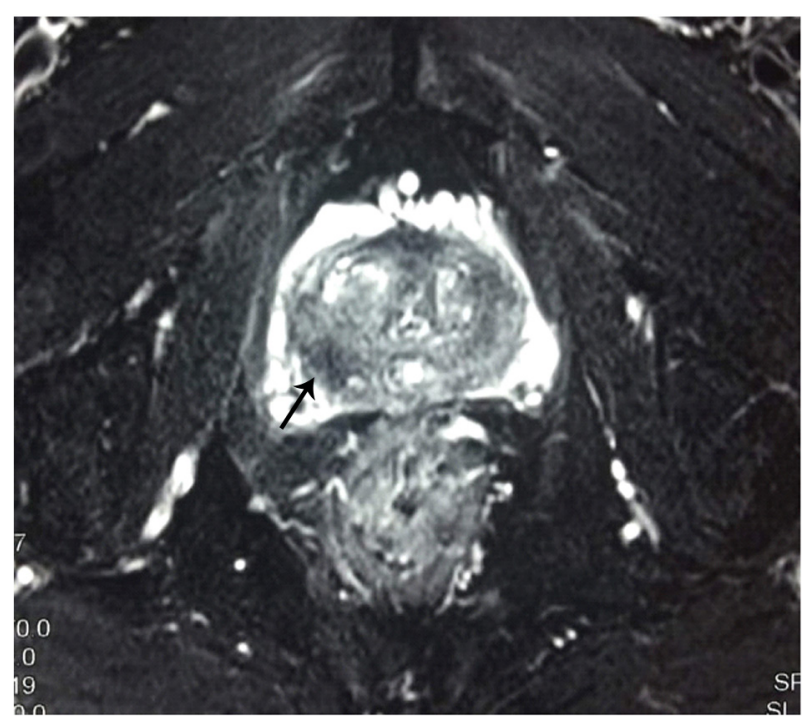

Figure 1. MRI showing T2 hypointense lesions in right peripheral zones of prostate (marked with arrows).
This was a prospective study conducted in the department of urology SKIMS Srinagar. The surgeries were performed between 2013 to 2020. Work up of the patients in the study $(n=42)$ included age, comorbidities, serum prostate -specific antigen levels, digital rectal examination, MPMRI prostate, prostatic biopsy (Gleasons score), bone scan and optional PSMA PET scan. In view of non availability of Robotic facility open surgery was performed. Intraoperative findings and pathological variables -T stage, nodal status, any extraprostatic extension, apical margin, bladder neck, seminal vesical invasion, lymph nodal status, post operative BCR need for any hormonal and salvage radiotherapy were recorded. On follow up particular emphasis was given on trifecta as cancer control, urinary continence, erectile function and overall satisfaction.

\section{Surgical Technique}

After the proper evaluation preoperative anesthesia clearances and preparation patients were planned for the surgical procedure. Procedure was done in supine position with 15 degree tilt at level of pelvis. All the procedures were done under epidural anesthesia there by avoiding the morbidity of general anesthesia. Infra umbilical midline incision was made. A standard pelvic lymphadenectomy was performed in all cases. Prevesical space was dissected, retropubic fat removed, endopelvic fascia opened ,puboprostatic ligaments transected, DVC sutures (Figure 2A, 2B) applied and DVC transected. Apical dissection (Figure 2) was done with utmost care to prevent any margin positivity, urethra transected and dissection carried in retrograde fashion. Neurovascular bundles when preserved were released from prostate without any undue traction during dissection and in those cases where neurovascular bundle seamed involved was resected enblock along with the specimen. Bladder neck was reconstructed (Figure 3) as per need using size 3 absorbable sutures. urethrovesical anastamosis (Figure 4) was done using eight, size 3-0 monocryl suture interrupted mucosa to mucosa absorbable sutures in watertight fashion over 18F silicon Foley's catheter. Pelvic drain was placed in all cases after completion of radical prostatectomy (Figure 5)

\section{Post operative care}

Postoperatively analgesia was administered with non opioid agents via epidural/intravenous route as per the standard post operative protocol followed in the hospital. After RPP the patients were ambulated on first postoperative day of surgery. The drain was removed once the patient passed flatus and 24 output was less than $100 \mathrm{ml}$. Patients were discharged with indwelling catheter once they were ambulatory, tolerating diet. Patients returned to outpatient department 21 days after surgery for catheter removal. Return of continence was assessed on day of catheter removal, at 1, 3,6 and 12 months after catheter removal. Patients were briefed about the process of estimation of extent of incontinence during their stay in the hospital itself, which was reinforced at the time of removal of catheter. On the day of catheter removal all patients were advised to wear an adult diaper. Depending upon the quantum of leakage of urine they were advised to change from the diaper to protection pads (underwear linings) and record the number of diapers/protective pads per day. Preoperative IIEF5 score were obtained after offering the IIEF-5 questionnaire to all the patients.All sexually active patients were started on penile rehabilitation programme using PD-5 inhibitors and erectile function was assessed at 6 and 12 months using the IIEF5 questionnaire. All postoperative specimens were examined by in-house pathologists at our institute. Details of histopathologic assessment were recorded including final Gleason score, margin 
Table 1. Clinical spectrum of patients $(N=42)$.

\begin{tabular}{|c|c|}
\hline Items & Data \\
\hline Age in years/ no. Of patients & $\begin{array}{l}50-59=15 \\
60-69=27 \\
\geq 70=0\end{array}$ \\
\hline Clinical staging & $\begin{array}{l}\mathrm{T} 1=\text { none } \\
\mathrm{T} 2(\mathrm{~T} 2 \mathrm{a} / \mathrm{T} 2 \mathrm{~b})=42 \\
\mathrm{~T} 3=\text { none }\end{array}$ \\
\hline Method of detection & $\begin{array}{l}\text { Incidental }=12 \\
\text { Screening }=5 \\
\text { Symptomatic luts }=25\end{array}$ \\
\hline Method of detection & $\begin{array}{l}\text { Incidental }=12 \\
\text { Screening }=5 \\
\text { Symptomatic luts }=25\end{array}$ \\
\hline Hypertension & 5 \\
\hline $\mathrm{T} 2 \mathrm{DM}$ & 3 \\
\hline PSA level (ng/dl) & $\begin{array}{l}4-10=9 \\
11-20=29 \\
>20=4\end{array}$ \\
\hline Gleson score on TRUS guided Prostate biopsy & $\begin{array}{l}3+4=28 \\
4+3=8 \\
4+4=6\end{array}$ \\
\hline Gleson score of radical prostatectomy specimen & $\begin{array}{l}3+4=22 \\
4+3=13 \\
4+4=7\end{array}$ \\
\hline Average blood loss & $460 \mathrm{ml}$ \\
\hline Positive apical margin & 2 \\
\hline Extracapsular invasion & 2 \\
\hline Bladder neck involvement & none \\
\hline Seminal vesical involvement & 2 \\
\hline
\end{tabular}

LUTS-Lower urinary tract symptoms.

positivity and seminal vesicle involvement and lymph node status. Postoperative PSA was assessed at 3 monthly intervals. Biochemical recurrence (BCR) was defined as two consecutive PSA level $\geq 0.2 \mathrm{ng} / \mathrm{ml}$ after RPP [8]. Satisfaction was evaluated by considering continence, cancer control, erectile function and cost as not satisfied, partially satisfied and completely satisfied.

\section{Results}

Mean age of patients in our study(Tabe 1) was 62 years ( range:55-68). Five patients were hypertensive and 3 patients were diabetic. Mean operative time was 180 minutes and mean blood loss was $460 \mathrm{ml}$. The average hospital stay of patients was 4 days. Patients were discharged with Foley's catheter in situ once drain was out and orals were adequately tolerated. Foley's Catheter was removed 3 weeks postoperatively after performing pericatheric study to look for any anastamotic leakage. Mean follow up was 40 months. On Histopathological examination (Table 1) 40 patients had T2 disease and 2 patients had seminal vesicle invasion. A positive surgical margin was seen in one patient at apical region. Out of 42 patients 7 patients were continent at 1 month follow up, 27 at 3 months, 39 at 6 months and 42 at 12 months. One patient continued to be incontinent at 1 year. Out of 16 patients with nerve sparing RPP, 10 patients were potent with PD 5 inhibitor assistance at 6 months and all at 1 year (Potency was defined as the ability to have erections adequate enough for penetration more than $50 \%$ of the times). Three patients had Biochemical recurrence on follow up and both were subjected to hormonal and salvage radiotherapy. Thirty eight patients were disease free at last follow up.

All the patients (41) were completely satisfied (Table 2) with 
Table 2. Number of pads used per day by patients after catheter removal/ continence status ( $\mathrm{N}=\mathbf{4 2})$ and overall satisfaction.

\begin{tabular}{|c|c|c|c|c|c|}
\hline $\begin{array}{l}\text { NO of Pads } \\
\text { Used Per Day }\end{array}$ & Day Zero & 1Month & 3Months & 6Months & 12Months \\
\hline No pad & 0 & 8 & 25 & 33 & 42 \\
\hline One pad & 0 & 6 & 13 & 6 & 0 \\
\hline Two pads & 2 & 15 & 3 & 2 & 0 \\
\hline Three pads & 25 & 7 & 1 & 1 & 0 \\
\hline Four pads & 13 & 2 & 0 & 0 & 0 \\
\hline Five pads & 2 & 0 & 0 & 0 & 0 \\
\hline Continence status & $0(0 \%)$ & $14(33 \%)$ & $38(90.4 \%)$ & $39(92.8 \%)$ & $42(100 \%)$ \\
\hline
\end{tabular}

Overall satisfaction at 1 year

respect to continence. Three patients with BCR were partially satisfied as they needed postoperative salvage radiotherapy and hormonal therapy. 16 patients were completely satisfied with erectile function at 1 year of follow up while in rest of the patients erectile function was not a concern and had opted for non nerve sparing procedure.

\section{Discussion}

Radical prostatectomy (RP) is a standard treatment for localized prostate cancer. The open radical prostatectomy with a retropubic approach was gold standard for many years. The first radical prostatectomy was performed by the perineal approach in 1903 by Young [4] and the first retropubic approach described by Millin [5] in 1947. The current concept of retropubic radical nerve-sparing prostatectomy was established by Patrick Walsh et al. in 1982 [6]. The next step in the evolution of open radical prostatectomies was transfer to a field of minimally invasive surgeries, i.e. laparoscopic radical prostatectomy (LRP) and robot-assisted laparoscopic radical prostatectomy. Oncological outcomes and positive margin rates (PMR) are equivalent between robot-assisted radical prostatectomy (RARP) and radical retropubic prostatectomy (RRP) [7]. The problem with robot-assisted radical prostatectomy is lack of availability and high peri operative costs. The equipments
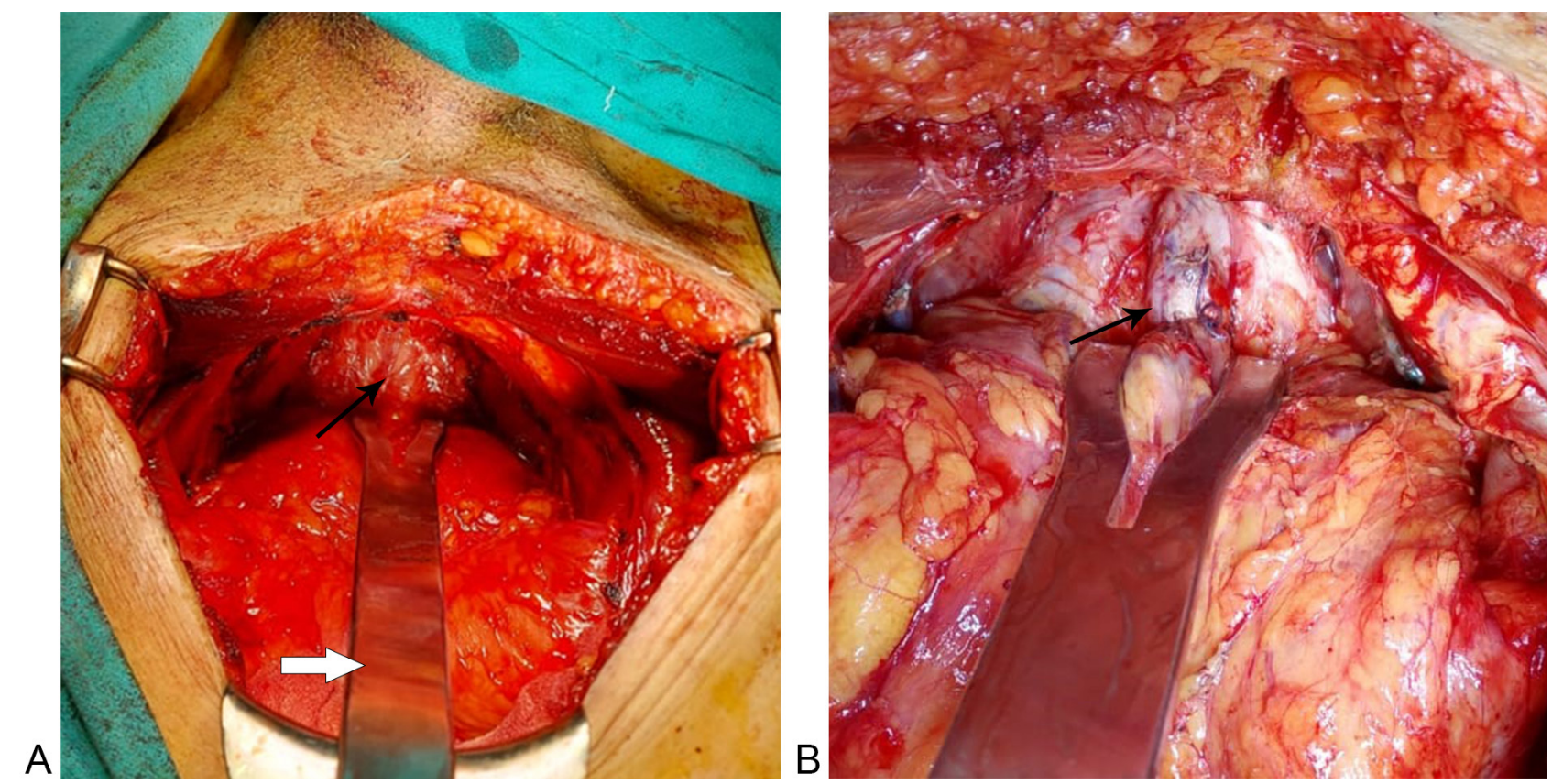

Figure 2A: Apical dissection in RPP for ligation of Dorsal venous complex(marked with arrow); Thick arrow showing apical retractor for apical dissection. 2B: Apical retractor with proximal control of Dorsal venous complex (marked with arrow). 


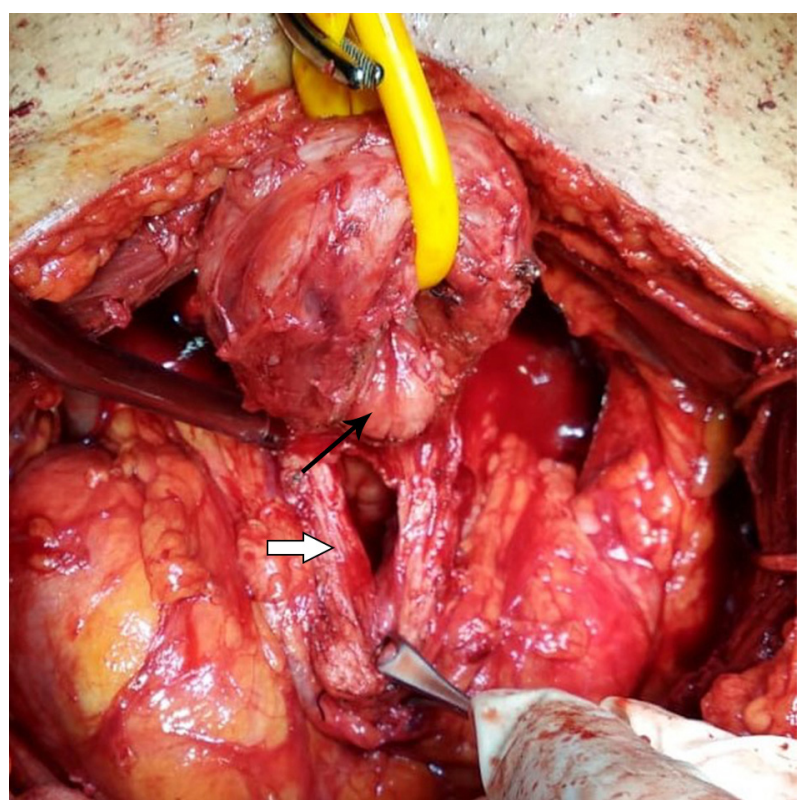

Figure 3. Completion of radical prostatectomy with median lobe (thin arrow) excision with opening of bladder neck (thick arrow).

are expensive and the operating time is, at least during the learning period, longer than in radical retropubic prostatectomy, while in laparoscopic assisted radical prostatectomy high degree of expertise is needed.

Although RRP is largely replaced by Robotic assisted Radical prostatectomy in developed countries, RPP continues to be the procedure of choice for carcinoma prostate in developing countries with limited resources and lack of Robotic technology. The advantages of RPP being that the procedure can be performed at any center with easily available armamentarium, and is very cost

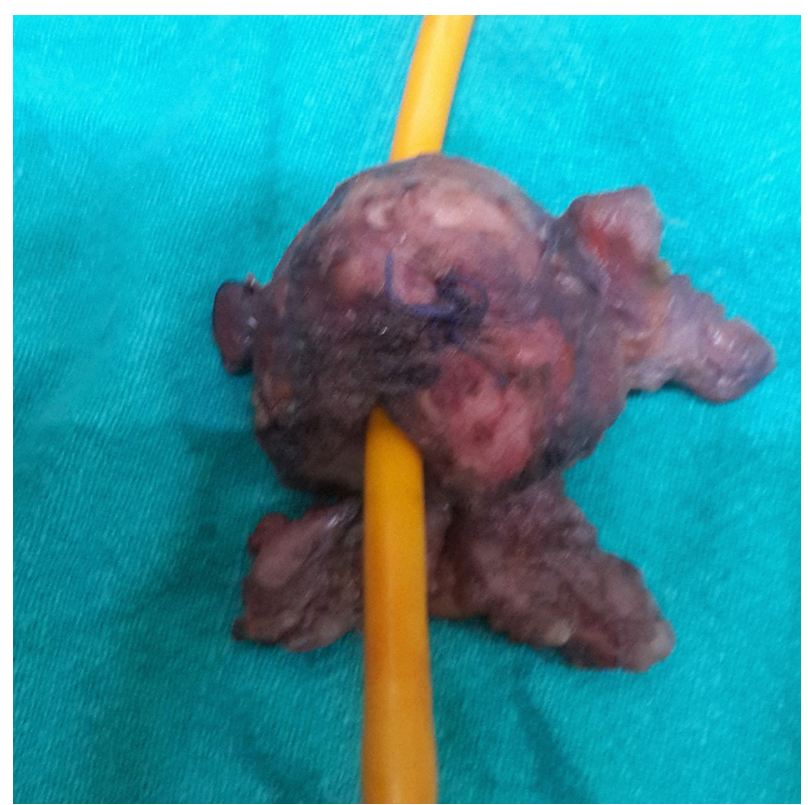

Figure 5. Completion of Radical prostatectomy specimen with bilateral seminal vesicles and vas in situ.

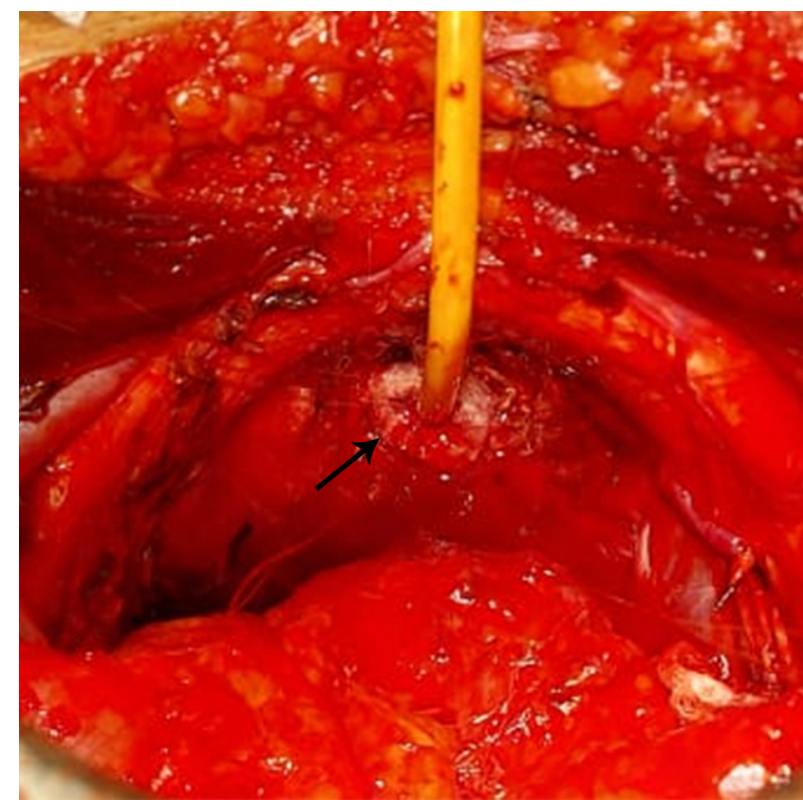

Figure 4. Urethrovesical anastamosis after RPP using size 3-0 monocryl absorbable interupted sutures (Marked with arrow).

effective. The infraumblical incision in RPP makes it less morbid and dissection is performed in extaperitoneal plane thereby no violation of peritoneal cavity. Urine leak/bleeding if occours is limited to extraperitoneum and lack of gut handling helps in the early return of bowel activity and thereby decreasing the hospital stay. Most of the procedures in our study were done under combined spinal-epidural anesthesia[9] which offers advantages of both spinal and epidural anesthesia; rapid onset and profound level of anesthesia, possibility of supplementary doses and postoperative pain management, enhancing the early postoperative recovery

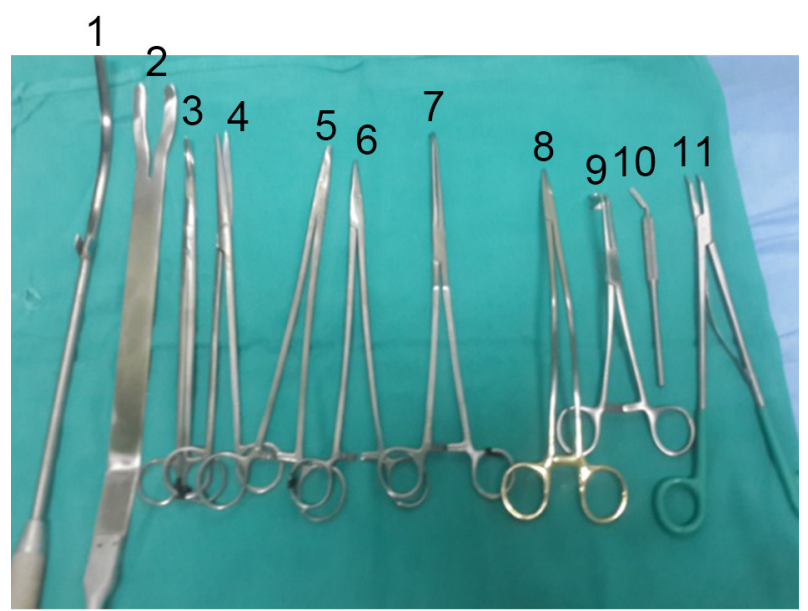

Figure 6. Specific open radical prostatectomy instruments. (1) Bladder neck retractor (2) Prostate Apical Retractor (3) Long curved scissors (4) Straight scissors $(5,6)$ Long Needle holders (7) Right angle holder (8) Curved needle holder (9) Right angle Babcock's (10) Cuved scapel for DVC Incision (11) Clip applicator. 


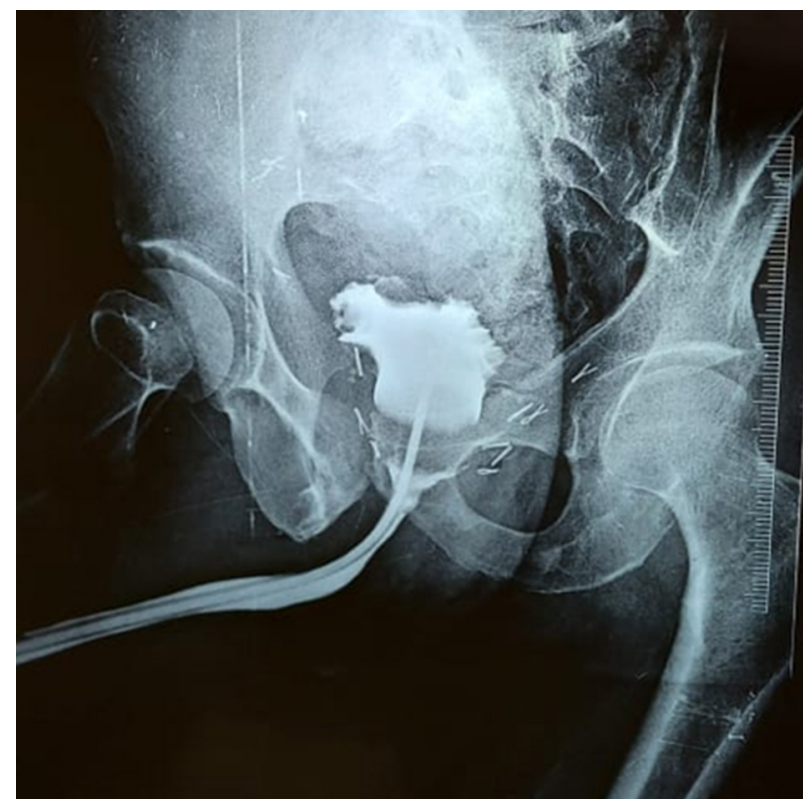

Figure 7. MCU before catheter removal at 3 weeks after RPP with no extravasation of contrast.

and obviating need for ventilatory support/ICU care. Another advantage of RPP with CSE anesthesia in the present COVID 19 era is avoiding need for sophisticated equipment needed for pneumoperitoneum to limit aerosol formation and no intubation, ventilation and extubation thereby reducing aerosol spread and exposure to health care personnel [10]. All patients preoperative imaging (Figure 1) as MPMRI Prostate were thoroughly discussed with the radiologist before surgery.

As far as the overall satisfaction is concerned at I year all the patients were completely satisfied with respect to continence. Two patients with BCR were partially satisfied as they needed postoperative adjuvant radiotherapy and hormonal therapy. 16 patients were completely satisfied with erectile function while in rest of the patients erectile function was not a concern and had opted for non nerve sparing procedure.

The instruments( Figure 6) which help in making the procedure easy improve better oncological outcome include Binocularloops, Apical retractor (Figure 2A) and bladder neck retractor which helps in better visualization of the planes and avoiding apical [11] and basal margin positivity respectively, Curved needle holder which eases in taking DVC control(Figure 2B) thereby reducing the blood loss. Postoperatively all patients were kept on low molecular weight heparin for deep venous thromboprophylaxis and PTE 12 hours after surgery and continued for 2 weeks postoperatively [12]. All the patients were continent at one year of follow up owing to meticulous dissection, preservation of urethral length as much as possible, pre and post operative pelvic floor exercises ( kegel exercises with concentration therapy) by hospital PMR department which has a significant impact on early recovery of continence after RPP [13]. MCU was done( Figure 7) in all patients before removal of Foleys catheter at two weeks to look for any anastomotic leak and if so catheter removal was delayed for another week to allow more time for the rent to heal. Urinary extravasation is supposed to induce inflammatory response which leads to stricture formation and delaying catheter removal helps in preventing the same. Six patients had intial extravasation of contrast which had settled on repeat MCU done at 3 weeks (Figure 7). All the patients voided after the removal of catheter with main complaint being incontinence which depends on age, intraoperative dissection, physical activity in the form of precise pelvic exercises and any associated patient factors as obese, and medical condition like Diabetes. Three patients in our study who had BCR were found to have focus of positive apical margin on histopathological examination and one patient had recurrence in prostatic fossa on PSMA PET CT, all these patients were subjected to adjuvant radiotherapy and hormonal therapy and responded well to the treatment. Patients were treated with normo fractionated intensity modulated radiation therapy (IMRT) at our centre to a dose of 70 Gy in 35 fractions. In all cases, volume contouring was done on a co-registered $3 \mathrm{~mm}$ MRI-CT fusion images. Clinical target volumes (CTV) and planning target volumes (PTV) included the entire tumor bed taking into account Radiation Therapy Oncology Group (RTOG) atlas. All surrounding normal structures were contoured to minimize dose absorption and keep constraints to an acceptable level. Step and shoot IMRT was planned on the Varian Eclipse software version 13.2 in all cases and delivered at our in-hospital Linac. Six months ADT was also added to these patients after completion of radiotherapy. Although robotic assisted radical prostatectomy is being most commonly performed in developed countries but as for as the trifecta of the surgery is concerned robotic as well as the RPP techniques have yielded equivalent effectiveness rates of functional, oncological, and surgical outcomes [14]. Infact many studies have shown RPP as a minimally invasive procedure that was found to be the most costeffective $[15,16,17,18]$. Success of open radical prostatectomy has improved with clear understanding of periprostatic anatomy.

Radical prostatectomy is the standard of care for organ confined prostatic carcinoma. Aim of the procedure is trifecta of cancer control, urinary continence, and erectile function. Minimally invasive techniques should not be a limiting factor especially when affordablity and non availability is concern. After open surgery such subset of patients enjoy good personal satisfaction with same oncological outcome as achieved by minimally invasive techniques. Furthermore it is the surgical expertise rather than technology that has a bearing on outcome especially when affordablity and non availability of robotic surgery is a factor.

\section{Conclusions}

Radical prostatectomy is the standard of care for organ confined prostatic carcinoma. Aim of the procedure is trifecta as cancer control, urinary continence, and erectile function. Minimally invasive techniques as Robotics should not be a limiting factor especially when affordablity and non availability is concern. After open surgery such subset of patients enjoy good personal satisfaction with same oncological outcome achieved by Robotics. Furthermore it is the surgical expertise rather than technology that has a bearing on outcome especially when affordablity and non availability of robotic surgery is a factor.

\section{Ethical policy}

All authors agree that there is no disclosure of potential conflict of intrest and research involving Human participants with informed consent from all enrolled patients in our study.

\section{Author contributions}

AR Khawaja: project development, Manuscript writing; MA Rouf: Data collection, Manuscript writing; YDar: Data collection; TMalik: Data collection; KSofi: Data collection; TMalik: Data collection; SA Malik: project development; AH Bhat: project development; MSWani: Project development Manuscript Editing.

\section{Competing interests}


The authors declare no competing interests.

\section{References}

1. Ferlay J, Shin HR, Bray F, et al: GLOBOCAN 2008 v 1.2, Cancer Incidence and Mortality Worldwide: IARC Cancer Base No. 10. Lyon, France: International Agency for Research on Cancer; 2010.

2. American Cancer Society: Cancer facts \& figures 2016. [cited 2016 May 16]. Available from: http://www.cancer.org/acs/groups/ content/@research/documents/document/acspc-047079.pdf .

3. Patel VR, Coelho RF, Chouhan S, Orvieto MA, Palmer KJ, Rocco B, et al: Continence, potency, and oncological outcomes after roboticassisted radical prostatectomy: early trifecta results of a high volume surgeon. BJU Int 2010; 106: 696-702.

4. Young HH: Conservative perineal prostatectomy: the results of two years' experience and report of seventy-five cases. Ann Surg 1905; 41: 549-557.

5. Millin T: Retropubic prostatectomy; a new extravesical technique; report of 20 cases. Lancet 1945; 2: 693-696.

6. Walsh PC, Lepor H, Eggleston JC: Radical prostatectomy with preservation of sexual function: anatomical and pathological considerations. Prostate 1983; 4: 473-485.

7. Abdollah F, Sood A, Sammon JD, Hsu L, Beyer B, Moschini M, et al: Long-term cancer control outcomes in patients with clinically high-risk prostate cancer treated with robot-assisted radical prostatectomy: results from a multi-institutional study of 1100 patients. Eur Urol 2015; 68: 497-505.

8. Cookson MS, Aus G, Burnett AL, et al: Variation in the definition of biochemical recurrence in patients treated for localized prostate cancer: the American Urological Association Prostate Guidelines for Localized Prostate Cancer Update Panel report and recommendations for a standard in the reporting of surgical outcomes. J Urol 2007; 177: 540-545.

9. Felsby, Sven MD, Juelsgaard, Palle MD: Combined Spinal and Epidural Anesthesia. Anesthesia \& Analgesia: April 1995 - Volume 80 - Issue 4 - p 821-826.

10. Stephanie N Morris MD, Amanda Nickles Fader MD, et al: Understanding the "Scope" of the Problem: Why Laparoscopy is Considered Safe During the COVID-19 Pandemic.Published:April0 3,2020DOI:https://doi.org/10.1016/j.jmig.2020.04.002.

11. Smith JA Jr, Chan RC, Chang SS, Herrell SD, Clark PE, Baumgartner R, Cookson MS: A comparison of the incidence and location of positive surgical margins in robotic assisted laparoscopic radical prostatectomy and open retropubic radical prostatectomy. J Urol. 2007 Dec; 178(6): 2385-9; discussion 2389-90. Epub 2007 Oct 22.

12. Felder S, Rasmussen MS, King R, Sklow B, Kwaan M, Madoff $\mathrm{R}$, Jensen C: Prolonged thromboprophylaxis with low molecular weight heparin for abdominal or pelvic surgery. Cochrane Database Syst Rev. 2019 Mar 27; 3: CD004318. doi: 10.1002/14651858. CD004318.pub4.

13. Kongtragul J, Tukhanon W, Tudpudsa P, Suedee K, Tienchai S, Leewansangtong S, Nualgyong C: Effects of adding concentration therapy to Kegel exercise to improve continence after radical prostatectomy, randomized control. J Med Assoc Thai. 2014 May; 97(5): 513-517.

14. Comploj E, Palermo S, Trenti E, et al: Radical perineal prostatectomy: An outdated procedure? Int J Surg 2011; 9(5): 400403.

15. Fu Q, Moul JW, Sun L: Contemporary radical prostatectomy. Prostate Cancer 2011:645030. Epub 2011 Apr 14.

16. Lepor H: Status of radical prostatectomy in 2009: is there medical evidence to justify the robotic approach? Rev Urol 2009; 11(2): 61-70.

17. Korman HJ, Harris MJ: Prostate Cancer - Radical Perineal Prostatectomy. Medscape.com. http://emedicine.medscape.com/ article/447239-overview.

18. Boccon-Gibod L: Radical prostatectomy: open? laparoscopic? robotic? Eur Urol 2006; 49 (4): 598-599. 